\title{
The Analysis of the Influence of the Reform of English Examination in College Entrance Examination on Some Students in Key High Schools in Shanghai
}

\author{
QiWang $^{1, a}$, YuwenDong ${ }^{1, b}$, LanHuang ${ }^{2, c}$ \\ ${ }^{1,2}$ Foreign Languages Department, East China University of Science and Technology, Shanghai, \\ P.R CHINA \\ accatherine_pwq@163.com, b448350194@qq.com, 'lan_huang@163.com \\ *Corresponding author: WANG Qi
}

\begin{abstract}
Keywords: English, College Entrance Examination Reform in Shanghai, learning styles,Essential-Qualities-Oriented (EQO) Education.
\end{abstract}

Abstract: The reform program of College Entrance Examination in Shanghai was issued in September 2014, which made a sweeping change in English examination. The Reform is intended to change the model of exam-oriented English education. This research focuses on the reform of English examination, the change of learning style and the progress of quality-oriented education. The independent-designed questionnaire about learning style and the progress of quality-oriented education were used to survey high school students in some key high school in Shanghai. The in-depth research was made on the basis of the analysis of data and the study of domestic and foreign researches. Finally, this research brings forth a conclusion: the fixed learning model hasn't been actually gotten rid of, and English study in high schools still focuses on doing a great amount of written exercise. However, the recent reform does pave the way for further promoting Essential-Qualities-Oriented (EQO) Education. In addition, it alleviates contradictions between gaining higher scores and acquiring more knowledge to some extent. The importance educators attach to English speaking and writing is increasing, occupying a similar part of time with English grammar in class. Students also hold active attitude towards English learning. In the meanwhile, according to the conclusion, researchers put forward some comprehensive and constructive suggestions on this issue.

\section{现行高考英语大型改革对于上海市部分重点高中学生 英语学习实际情况的影响分析 \\ 王琪 ${ }^{1, a}$ ，董玉文 ${ }^{1, b}$ ，黄岗 ${ }^{2,0}$ \\ 1,2 华东理工大学外国语学院英语系, 上海, 中国 \\ accatherine_pwq@163.com, b448350194@qq.com, 'lan_huang@163.com \\ *通讯作者: 王琪}

关键词：英语；上海高考改革；学习方式；素质教育

摘要： 2014 年 9 月出台的上海高考改革方案, 对英语考试进行了大规模的改动。旨在改变英 语教育长期以应试教育为主的模式。本研究重点关注高考英语改革, 对学生学习方式的转变 以及对素质教育的实际推动情况。通过自主设计的关于学习方式和素质教育情况的调研问卷, 数据统计并结合国内外研究成果, 进行详细分析, 对改革的落实情况进行了深入的研究。最 终得出结论: 未能摆脱长期以来英语学习的固化模式, 英语学习所需时间普遍较长, 且集中于 笔头练习部分。但本次改革为进一步推进素质教育开辟了道路，在一定程度上缓解当前应试 与教育的矛盾, 对口语和写作等英语素质的重视程度有所提高, 与语法部分相当, 学生对于 
英语学习热情得以肯定。研究者也在此基础上对此次英语改革提出了相关的意见和建议。

\section{1. 研究背景}

高考作为中国特色的考试制度, 发挥着选拔人才、发展高等教育重要作用。但是, 这一 制度的存在, 导致中国教育长期畸形发展。因此 2014 年 9 月, 英语高考改革应运而生。上海 市人民政府正式出台了《上海市深化高等学校考试招生综合改革实施方案》。新方案规定, 2017 年起, 外语考试由单纯笔试转变为笔试和听说测试相结合。

而我们针对英语高考改革后的学习方式变化和素质教育情况开展深入的调查和研究，力 争揭示改革对英语教育的影响。因此, 我们的研究具有鲜明的时代意义, 能够对改革成效进 行一定程度的评估, 并对未来英语教育以及英语高考改革提供合理意见。

\section{2. 国内外研究现状}

国内外教育界, 对英语学习方式以及英语素质教育已经展开过深入研究, 为我们进行进 一步的探究提供了广泛而坚实的理论基础。

在国外, 素质教育开展时间较长, 为我国英语素质教育提供借鉴。美国学校中, 素质教 育与相应的教学体制相结合, 注重培养学生的自主性。日本学校, 重视个性发展为原则, 发 展青少年的个性和能力倾向, 培养学习热情、创造能力和判断能力 ${ }^{[1]}$ 。

在国内, 针对素质教育及学习方式的研究也在大规模开展。首先, 素质教育核心目的在 于促进人的全面发展 “它规定着把受教育者培养成什么样人的根本问题, 是教育活动的出发 点。” ${ }^{[2]}$ 其次, 对英语素质教育学术界也有了深入研究, 英语素质教育作为英语文化素质 “要 求学生全面掌握英语的听、说、读、写等基本技能，并且能够用英语进行日常交际同时了解 一定的重西方文化差异以及具有自主学习英语的能力。” ${ }^{[3]}$ 旨在构建 “培养创新精神和实践能 力的学习方式, 倡导学生为主体教师为主导的探究性学习, 促进合作化学习和开拓自主型学 习” ${ }^{[4]}$ 可见英语素质教育已经成为大势所趋, 转变学习方式也成为现阶段教育的重点。

\section{3. 本文研究方向}

综合上述研究，我们发现，国内外都对素质教育以及教育改革下学生的学习方式转变给 予了极大重视和充分研究, 也都为本项目研究提供了充足的理论基础。但也存在着一些不足。

第一，时代在不断变化，部分研究成果受到时间限制，无法对当前状况进行充足有力的 说明。第二, 部分结果以西方素质教育为背景, 存在着与我国国情不相适应的部分。第三, 大部分研究成果以教师教学为主要出发点, 缺乏对学生本身的研究和分析。

因此，我们立足于上海高考改革新政策，通过设计问卷，对学生进行深入分析，探究在 高考改革后, 英语学习方式和素质教育情况的转变, 进行更加具有创新性的分析。通过在上 海市大同中学、上海市青浦高级中学、上海市奉贤中学、上海市民立中学共四所实验性示范 性高中, 对共计两百余名师生进行相关的问卷调查, 并且结合国内外素质教育特点, 得出了 初步分析结果，最终对高考改革下的英语学习方式和素质教育的未来发展方向提出建议。

\section{4. 数据分析}

\section{1 课下学习}

教育部 2001 年颁发的《基础教育课程改革纲要（试行)》提出要逐步实现学生学习方式 变革 ${ }^{[5]}$ 。英语高考改革, 旨在转变以应试教育主的现行教育模式, 真正提高学生的英语综合 素质和能力。而课下英语用时分配能够在很大程度上反映英语学习的主要方式和侧重点。

学生的英语家庭作业主要以书面练习为主, 通过大量练习, 以提高英语应试技巧。通过 
数据收集与统计可以得出, 英语课下家庭作业仍然占用大量的时间。在用时方面, 作业时间 占用 60 分钟以上的达总人数的 $59.4 \%$ 。

而阅读英文书籍作为提升英语素养的有效途径, 其用时能够在一定程度上反映出, 学生 对英语综合素养培养的重视程度。但通过调查研究, 可以看出, 英语素质教育仍没有引起足 够重视。在用时方面, 接近 70\%的学生的课外英语阅读时间在 30 分钟以下, 而 45 分钟及以 上的人数不足总人数的 $8 \%$ 。

将两者用时进行比较, 可以得出两个结论。一方面, 英语高考改革后, 学生实际英语学 习方式未发生转变, 未能摆脱传统的应试学习模式, 应试学习方式仍是主流。另一方面, 从 极值来看, 英语素质教育情况也不容乐观。两组数据中, 最大值出现在课外阅读三十分钟以 下所占人数, 数值接近 $70 \%$, 比次高值即课下英语作业用时 60 分钟以上的 $60 \%$ 还要高出 $10 \%$ 。

\section{2 课堂学习}

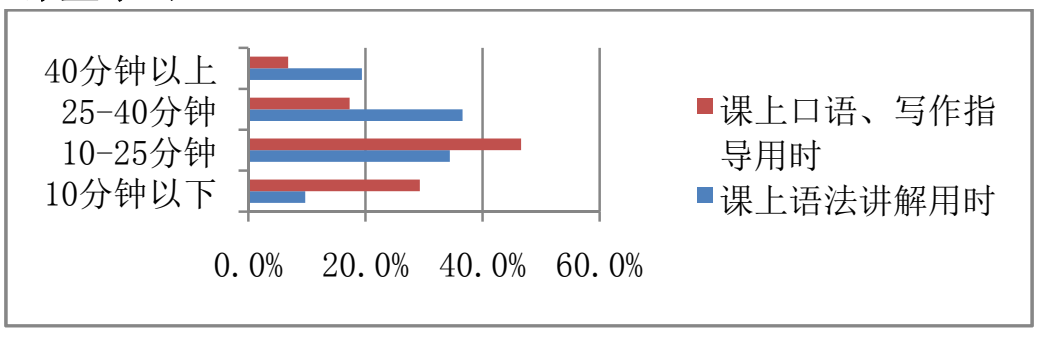

图 1 课堂学习时间分布百分比

课堂学习是英语教育的重要环节, 课堂内容的时间分配能够在很大程度上体现英语教育 的重点。传统的英语教学重视词汇、语法的教学, 教师一味地灌输、学生被动地听讲。这样 的教学方式没有充分体现语言的实践性特征 ${ }^{[6]}$ 。

语法作为可以书面量化考察的内容, 一直是应试教育关注的核心, 其课堂所占时间较多。 由图 1 可以看出, 课堂语法讲解用时占用 10-25 分钟的学生占总人数的 34. 4\%, 占用 25-40 分钟的共占总人数的 $36.6 \%$ 。而口语、写作作为重要的英语能力, 其课堂所占时间较少。

通过两组数据对比, 我们可以得出: 首先, 英语课堂教学内容仍以应试为主。接近 $40 \%$ 的学生其英语课堂总时长的一半以上用于讲解英语语法。接近 50\%的学生仅有不到 $1 / 5$ 的课 堂时间用于提高口语和写作。其次, 素质教育重视程度不均, 课堂上用于指导口语和写作的 时间在两组数据中极差最大, 接近 $40 \%$, 波动较大, 以素质教育为育人之本的理念并没有得 到贯彻。

\section{3 主观想法}

根据问卷调查的结果显示, 有近 $65 \%$ 的中学生认为, 英语学习水平的提升需要实际的演 练环境, 而这恰恰也是目前中国英语教学最为缺乏的部分。上海市《中长期教育改革和发展 规划纲要 (2010-2020 年)》明确提出, 要 “试点开设高中国际课程, 鼓励有条件的中小学开 设由外籍教师执教的课程” ${ }^{[5]}$ 。根据我们的调查采访, 此类课程占比时间极少, 半个月才能 上到 45 分钟。这与某些学校国际部, 逾半数教师都是外教相比, 可谓差距甚远。

那么, 聘请外教进行教学是否对于学生有所助益呢? 在一项有关外国教师教学实效性的 探究中 ${ }^{[6]}$, 作者赵莉莉发现, 外教的幽默教学方式引起了学生对于西方文化的兴趣, 提高了 英语的学习动力, 在一定的程度上提高了学生的英语水平。据其调查显示, 83\%的学生听、说 能力有明显地提高。但是, 外教教学也存在了很多不足之处, 例如由于中西方文化的差异等。 来自定西市第一中学的卢慧珍 ${ }^{[7]}$ 也指出, 外籍教师往往教学资历和经验不足, 水平参差不齐, 容易存才误导学生的隐患。因此, 如何更好地满足学生英语学习主管需要的实际演练环境, 是我们所要重点关注和研究的。

\section{4 满意程度}

根据我们抽查的数据来看, 虽然选择 “不满意” 的同学占多数, 达到总数的 $54.8 \%$, 但 
也仅仅略占优势，剩下近半数的高中生则认为现在学习到的知识切实符合他们的主观期待。 从这道简单的 “判断题” 中我们可以得出, 目前上海市的英语教育仍有许多可取之处, 盲目地反对颠覆原有的英语教育模式是错误的。“教育以学生为本, 是教育的一个永恒的主 旨。 ${ }^{[8]}$ ” 所有的教育改革, 都必须以服务学生, 有利于学生的成长发展作为落脚点与核心。 只有这样的改革才是正确的。就教育根本目的而论, 就在于提高人的素质, 从而提高人的地 位, 具体就是要发现人的价值, 发掘人的潜能, 发展人的个性以及发挥人的力量 ${ }^{[9]}$ 。

\section{5 学习期望}

根据学生们自由选择自己最想要学习到的英语知识来看, 其中 “实际英语交流能力” 占 到了绝对的优势, 有 $83.5 \%$ 的学生渴望增长这一方面的知识储备。另外, 选择词汇、文化传 统和名著历史的同学也都超过了半数。

总体可以看出, 学生们对于多方面英语知识的期待基本上都是积极向上的。然而, 也有 学者发现, 虽然教材内容有过变动, 但是教学情况仍不如预期。上海师范大学的孙美华 ${ }^{[10]}$ 发 现, 在部分上海市实验性示范性高中里, 实际教学依旧偏重语言知识和技能, 对于文化等所 谓 “与考试无关”的知识则选择性地进行教授，灵活性很大，规范性不够。

由此得出, 目前英语教育中, 我们对于学生 “想要学什么” 的问题不够看重。在英语教 育中, 一定要关注学生主体的意见, 不能一味地应试, 更要教授他们感兴趣的内容, 引起学 生的学习动力和潜力, 这样才能真正做好教育。

\section{5. 研究总结}

通过本小组对于多所上海市实验性示范性高中学生的抽样调查, 加以一定的数据统计分 析比对, 在已有的理论基础上, 初步总结出了本次高考英语大型改革对于实际英语学习方式 改变以及素质教育情况的结论。

第一，英语学习所需时间普遍较长，且集中于笔头练习部分。上海市英语教材编写以及 考试政策近年来一直有所细微的调整与变化, 其最终目的与期许仍然是希望可以帮助学生学 习到更多实用的知识, 发展自主学习和合作学习的能力, 形成有效的英语学习策略, 培养学 生的综合语言运用能力 ${ }^{[11]}$, 帮助他们脱离 “应试教育”、“题海战术” 以及 “死记硬背” 的 弊端。然而, 根据本小组成员的抽样调查数据显示, 学生完成英语家庭作业的时间要远超于 阅读课外英语书籍的时间，整体的英语学习重点仍然处于 “做习题”上。

“在普遍的快教育模式下，中小学生进入了分数的生产线，….., 为了克服遗忘或者说 是减少遗忘, 只能是重复训练。” ${ }^{[12]}$ 这种训练动物时使用的高强度重复训练, 直接导致学生其 实是在 “背诵” 而非 “掌握” 知识点, 更不用说受到文化 “熏陶”了。但是, 学习一门语言, 除了需要一定的词汇量作为基础以外, 名人名著的阅读, 好词好句的积累, 才是真正助益于 学生内涵修养、品格健全的部分。“全面推进素质教育, 是我国教育事业的一场深刻变革, 是一项事关全局, 影响深远和涉及社会各方面的系统工程” , 并且需要 “拓宽人才成长的道 路, 减缓升学压力” ${ }^{[13]}$ 因此, 英语教育改革, 仍旧远远没有达到理想效果, 需要进一步的研 究与发展。

第二, 改革之后口语和写作的时常有所增加, 与语法部分相当。这相对之前缺少重视或 缺少有效教学方式的现象 ${ }^{[14]}$ 相比, 有了极大的改观。这一点, 是由此次英语高考形式大型变 革所带来的值得肯定的变化。

其三，学生对于英语学习的期待和热情得以肯定。参与抽样调研的部分上海市实验性示 范性高中学生, 主观上对于英语的学习, 多数持着积极向上的态度。无论是对于英语文化知 识、交流技能的期待, 还是对于文学名著、历史发展的渴求, 这些高中学生们对于英语学科 都抱有着热情。这一项十分令人欣慰的结果。只有使高中学生的主观能动性充分发挥, 学习 热情充分高涨, 才能让这些 “半大人” 们真正热爱学习。 ${ }^{[15]}$ 因此, 既然学生们对于英语学科 的学习充满着期待与渴望, 英语学习方式和改革就应该朝着学生需求的方式进行改变, 从而 
真正做到 “在快乐中学习与成长”。

\section{致谢}

本文为上海市级 “大学生创新创业训练计划” 项目《高考英语大型改革对于上海部分重 点高中学生及老师的影响实证研究》的阶段性成果之一。

\section{References}

[1]Zhang Yuming. Preliminary Exploration on International Essential-qualities-oriented Education- Its Features and Revelation for China [J]. China Education Innovation Herald, 2011, 35:25.

[2]Ma Jiansheng. Modern Education System and Thoughts [M]. Beijing: Higher Education Press, 2004:25.

[3]Nie Haitang. Brief Analysis onEssential-qualities-oriented Education at High School Level. Learning Weekly: Mid, 2016, 0 (3); 77-77.

[4]Liu Quanjin. The Study on Changing Students' Study Methods [D].Central China Normal University, 2007.

[5]Liu Daoyi. The Approach to Optimizing Methods of High-school English Study [J].Foreign Language Teaching in Schools, 2006, 08: 1-6.

[6]Yu Cuiye. High-school English Teaching Should Focus on Humane Quality Education- The Exploration on High School English Curriculum Standards (Tests) [J]. Journal of Basic English Education, 2004, 02: 43-49.

[7]Wang Fang. Comments on the Development of High-school International Courses in Shanghai [J]. Shanghai: Basic Education, 2012-8: 68-73.

[8]Zhao Lili. The Exploration on the Efficiency of Foreign Teachers' Teaching under the Principle of Newly-issued High School English Curriculum Standards [J]. Hebei: Xue Yuan Jiao Yu, 2012-2: 89.

[9]Lu Huizhen, The Strength and Weakness of Foreign Teachers'English Teaching in High Schools [J]. Hebei: Research in Teaching, 2010 (31): 304.

[10]Liao Wensheng, Opinions on Student-oriented Education [J]. Heilongjiang: Jiao Shu Yu Ren, 2014-2: 10-11.

[11]Yan Puyuan. Student-oriented Education- the Reflections on Modern Education in Schools in China [D]. Guangzhou: SUN YAT-SEN UNIVERSITY PRESS, 2005: 240.

[12]Sun Meihua. The Study on Background Knowledge in Textbooks of High-school English [D]. Shanghai: Shanghai Normal University, 2011.

[13]Ding Yuansheng. Thoughts on How to Deal with Curriculum Reform of High-school English [J].Ren Min Jiao Shi Lun Tan, 2009 (8): 8- 10.

[14]Cheng Pingyuan. Survey on Issues of China's Education [D]. Beijing: Tsinghua University Press, 2013: 163-164.

[15]CPC Central Committee and State Council on Deepening Education Reform to Promote Quality Education Decision [J].Hubei Education, 1999 (Z9): 4-8.

[16]Cheng Xiaowei. Brief Analysis on Teaching High-school Oral English [J]. Learning Weekly, 2011 (29): 167-167.

[17]Zhang Xinling. Opinions on the Inspiration of English-learning Enthusiasm of High School Students [J].New Education Era, 2015 (32). 